\title{
CRESCIMENTO E DESEMPENHO ECONÔMICO DE Eucalyptus dunnii EM DIFERENTES SÍTIOS NO PLANALTO SUL DO BRASIL
}

\author{
Mário Dobner Jr. ${ }^{1}$, Kamila Mesquita Batista ${ }^{1}$, Ian Pereira Sartório ${ }^{2}$, Julio Eduardo Arce ${ }^{3}$, \\ Dagoberto Stein Quadros ${ }^{4}$ \\ ${ }^{1}$ Universidade Federal de Santa Catarina, Curitibanos, Santa Catarina, Brasil - mario.dobner@ufsc.br; kamilamesquitab@gmail.com, \\ ${ }^{2}$ Florestal Gateados Ltda., Campo Belo do Sul, Santa Catarina, Brasil - ian@gateados.com.br, \\ ${ }^{3}$ Universidade Federal do Paraná, Curitiba, Paraná, Brasil - jarce@ufpr.br, \\ ${ }^{4}$ Universidade Regional de Blumenau, Blumenau, Santa Catarina, Brasil - dagobert@ furb.br
}

Recebido para publicação: 06/03/2016 - Aceito para publicação: 26/10/2017

\begin{abstract}
Resumo
A busca por maior produtividade, menores ciclos de produção e, consequentemente, maior desempenho econômico tem levado empresas e proprietários rurais do Planalto Sul do Brasil a investir em plantações de Eucalyptus em detrimento de Pinus. A determinação da produtividade e do desempenho econômico de povoamentos florestais é fator básico para o planejamento e otimização do manejo destes. Nesse contexto, o presente estudo foi realizado com o intuito de avaliar o crescimento e o desempenho econômico de plantios de Eucalyptus dunnii em diferentes sítios no Planalto Sul do Brasil. Foram utilizados dados de inventário florestal contínuo, totalizando 128 unidades amostrais e 10.903 pares de dados dap $\mathrm{x} \mathrm{h}_{\mathrm{dom}}$. Foram obtidas três classes de sítio com alturas dominantes assim distribuídas na idade índice de 10 anos: Classe I: 34 a 39 m; Classe II: 29 a 33,9 m; Classe III: 24 a 28,9 m. Povoamentos com d $\mathrm{d}_{200}$ de $30 \mathrm{~cm}$ podem ser obtidos já aos 9 anos de idade em sítios altamente produtivos. Entretanto, demoram de 3 a 5 anos a mais para serem obtidos em sítios menos produtivos. A taxa interna de retorno (TIR) de sítios altamente produtivos é duas vezes maior que em sítios de menor produtividade. Valor presente líquido (VPL) e valor esperado da terra (VET), no sítio de alta produtividade, são 14 vezes superiores aos observados nos piores sítios. Conclui-se não só que é mais rentável produzir $E$. dunnii em sítios mais produtivos, mas também que o cultivo de $E$. dunnii pode não ser viável em sítios pouco produtivos.

Palavras-chave: Manejo Florestal; Eucalipto; manejo para multiprodutos.
\end{abstract}

\begin{abstract}
Growth and economic performance of Eucalyptus dunnii at different sites on the highlands of southern Brazil. The search for greater productivities, shorter production cycles and thus higher economic performances have led companies and landowners on the Highlands of Southern Brazil to invest in Eucalyptus plantations rather than Pinus. Determining the productivity and economic performance of forest stands is a key factor for their planning and management. In this context, the present study was carried out aiming to evaluate de growth and economic performance of Eucalyptus dunnii in different sites on the Highlands of Southern Brazil. Data from continuous forest inventory were regarded with a total of 128 sample plots and $10.903 \mathrm{dbh} \times \mathrm{h}_{\text {dom }}$ paired values. Three site classes were obtained with dominant heights at index age of 10 years as the following: Class I: 34 to 39 m; Class II: 29 to 33,9 m; Class III: 24 to 28,9 m. Stands of $\mathrm{d}_{200}$ of $30 \mathrm{~cm}$ can be obtained since the age of 9 years-old on sites of high productivity, while it takes from 3 to 5 years on sites of lower productivity. The internal rate of return (IRR) on sites of high productivity is twice as high as the ones verified on sites with lower productivity. Net present values (NPV) and land expectation values (LEV) on sites of high productivity are 14 times higher than the ones observed on the worst sites. It was concluded that it is not only more profitable to grow E. dunnii on sites of high productivity, but also that there might be no viability at all of its cultivation on low productivity sites.

Keywords: Forest Management; Eucalypt; Multiproducts Management.
\end{abstract}

\section{INTRODUÇÃO}

A área reflorestada no Brasil com o gênero Eucalyptus é superior a 5 milhões de hectares (ABRAF, 2013). Segundo Alfenas et al. (2009), as diferentes espécies de Eucalyptus cultivadas no país possuem crescimento entre 30 e $80 \mathrm{~m}^{3} \mathrm{ha}^{-1} \mathrm{ano}^{-1}$. Esse elevado potencial de crescimento é um dos principais responsáveis pela extensa área cultivada com este gênero no país.

A busca por maior produtividade, menores ciclos de produção e, consequentemente, maior desempenho econômico tem levado empresas e proprietários rurais do Planalto Sul do Brasil a investir em plantações de 
Eucalyptus em detrimento de Pinus, o gênero mais cultivado nesta região. Entretanto, não raramente, observamse povoamentos de Eucalyptus com péssimo desempenho, aquém de plantios de Pinus. Isso ocorre devido à elevada exigência do gênero com relação ao sítio. De acordo com Scolforo (1997), o sítio é a expressão de todas as influências biológicas e ambientais no crescimento das árvores.

Eucalyptus dunnii Maiden, por ser uma espécie subtropical, tem se destacado no Planalto Sul do Brasil pelo rápido crescimento, uniformidade dos talhões, forma das árvores e moderada resistência à geadas (HIGA, 1998; HIGA et al., 2000; PALUDZYSZYN; DOBNER JR. et al., 2009). Além do Sul do Brasil, E. dunnii tem sido amplamente cultivado no Uruguai (HERNANDEZ et al., 2009), Argentina (MARCÓ; WHITE, 2002), China (ARNOLD et al., 2004) e Austrália (GLENCROSS et al., 2011).

A principal utilização da madeira de E. dunnii tem sido o abastecimento de indústrias de celulose, carvão vegetal, chapas de partículas e fibras; entretanto, sabe-se do seu potencial para usos sólidos mais nobres (HARWOOD, 2005; ROCHA; TRUGILHO, 2006; GLENCROSS et al., 2011). Em Santa Catarina, observa-se um grande consumo de madeira de E. dunnii para a produção de energia nos mais diversos setores da economia.

Independente dos objetivos da produção, a determinação da produtividade e do desempenho econômico de povoamentos florestais é fator básico para o planejamento e otimização do manejo destes. Nesse contexto, o presente estudo foi realizado com o intuito de avaliar o crescimento e o desempenho econômico de plantios de Eucalyptus dunnii em diferentes sítios no Planalto Sul do Brasil.

\section{MATERIAL E MÉTODOS}

Os povoamentos de E. dunnii avaliados apresentavam idades entre 3 e 24 anos, com maior intensidade amostral entre 6 e 11 anos. Apesar do manejo não ser objeto de análise, todos os povoamentos foram submetidos a regimes muito semelhantes que consistiram no plantio de 1.600 árvores ha $^{-1}$. Um primeiro desbaste foi realizado aos 4 anos de idade, removendo, aproximadamente, 50\% do número de árvores; um segundo desbaste, também de intensidade de 50\%, no sexto ou sétimo ano e um último desbaste por volta dos 9 a 10 anos de idade, reduzindo a densidade por hectare para cerca de 200 árvores. O corte raso desses povoamentos é previsto para os 20 anos de idade.

Os povoamentos de onde os dados foram coletados estavam localizados nos municípios de Campo Belo do Sul e Capão Alto (SC), entre as coordenadas $50^{\circ} 45^{\prime}$ e $51^{\circ} 05^{\prime}$ Longitude Oeste, e $27^{\circ} 35^{\prime}$ ' e $28^{\circ} 05^{\prime}$ Latitude Sul, com elevação média de $970 \mathrm{~m}$ s.n.m. A temperatura média anual da região é de $16^{\circ} \mathrm{C}$; a média mínima de $4,8^{\circ} \mathrm{C}$, a média máxima de $28,4^{\circ} \mathrm{C}$ e a mínima absoluta de $-6^{\circ} \mathrm{C}$ (EPAGRI/CIRAM - dados não publicados).

Foram utilizados os seguintes dados de inventário florestal contínuo, coletados entre os anos de 2011 e 2014: diâmetro a altura do peito (DAP, em $\mathrm{cm})$, altura total $(\mathrm{H}, \mathrm{em} \mathrm{m})$ de pelo menos 30 árvores por projeto e ano de plantio, e altura dominante $\left(\mathrm{H}_{\mathrm{dom}}\right.$, em $\left.\mathrm{m}\right)$. As alturas foram amostradas de acordo com o método de Assmann: a altura média das 100 árvores de maior dap ha ${ }^{-1}$. Os dados foram obtidos em parcelas circulares com área de $500 \mathrm{~m}^{2}$, remedidas anualmente durante o inverno. Ao todo, foram medidas 128 unidades amostrais, nas quais 10.903 pares de dados dap $\mathrm{x}_{\mathrm{dom}}$ foram coletados.

\section{Análise do crescimento}

Os dados de inventário florestal foram utilizados para o ajuste de modelos de crescimento. O primeiro passo foi determinar o crescimento em altura dominante $\left(\mathrm{h}_{\text {dom }}\right)$, permitindo o estabelecimento de 3 classes de sítio, a partir do método da curva guia e considerando a amplitude dos dados observada na idade índice (10 anos). Após a determinação das classes de sítios, as unidades amostrais foram classificadas e agrupadas de acordo com a respectiva capacidade produtiva. Esse agrupamento por classe de sítio antecedeu as análises de crescimento em diâmetro e volume.

Para a análise do crescimento em diâmetro, considerou-se a média das 200 árvores mais grossas por hectare $\left(\mathrm{d}_{200}\right)$, pois esta é a densidade final considerada ideal para um povoamento manejado para múltiplos usos. Dessa forma, avaliou-se o desenvolvimento médio das árvores objeto do manejo, ou seja, aquelas que permanecem no povoamento até o corte final. Essa análise foi realizada com base em 1.280 pares de dados $\mathrm{d}_{200} \mathrm{x}$ idade. Os mesmos dados permitiram, ainda, a avaliação do desenvolvimento do volume médio das árvores $\left(\mathrm{v}_{200}\right)$.

O volume individual das árvores foi obtido por meio do modelo de Schumacher e Hall (1933), ajustado para a espécie e região estudada pela empresa detentora dos povoamentos.

$$
\begin{gathered}
\ln \left(v_{i}\right)=-9,77463+1,92778(\ln \text { dap })+0,91743(\ln h) \\
R^{2}=0,99, S_{y x}=0,07
\end{gathered}
$$


Para estimar os volumes individuais, foi necessário estimar as alturas que não foram medidas nos inventários florestais. Essas alturas foram estimadas com equações hipsométricas ajustadas ano a ano a partir do modelo parabólico proposto por Trorey (1932).

Foram testados quatro modelos para o ajuste de curvas de crescimento das variáveis $\mathrm{h}_{\text {dom }}, \mathrm{d}_{200} \mathrm{e} \mathrm{v}_{200}$. Esses modelos são apresentados na Tabela 1. Além disso, as estimativas obtidas com os modelos linearizados (ln) foram corrigidas pelo Fator de Meyer, em função da discrepância logarítmica. A precisão do ajuste dos diferentes modelos foi avaliada a partir das respectivas estatísticas: coeficiente de determinação ( $\left.\mathrm{R}^{2}\right)$, erro padrão da estimativa $\left(\mathrm{S}_{\mathrm{yx}}\right)$ e análise gráfica dos resíduos.

Tabela 1. Modelos matemáticos testados para o ajuste de equações de crescimento de $h_{\text {dom }}, d_{200}$ e $v_{200}$.

Table 1. Mathematical models tested for fitting $\mathrm{h}_{\mathrm{dom}}, \mathrm{d}_{200} \mathrm{e}_{200}$ growth equations.

\begin{tabular}{cc} 
Modelo & Forma de Ajuste \\
\hline Polinomial & $\mathrm{y}=\beta_{0}+\beta_{1}(\mathrm{Id})+\beta_{2}\left(\mathrm{Id}^{2}\right)+\varepsilon$ \\
Hiperbólico & $\mathrm{y}=\beta_{0}+\beta_{1}\left(\frac{1}{\mathrm{Id}^{2}}\right)+\varepsilon$ \\
Potência & $\ln (\mathrm{y})=\beta_{0}+\beta_{1} \ln (\mathrm{Id})+\varepsilon$ \\
Exponencial & $\ln (\mathrm{y})=\beta_{0}+\beta_{1}\left(\frac{1}{\mathrm{Id}}\right)+\varepsilon$ \\
\hline Em quee: $\mathrm{y}=\mathrm{h}_{\mathrm{L}}$ &
\end{tabular}

Em que: $\mathrm{y}=\mathrm{h}_{\mathrm{dom}}, \mathrm{d}_{200}, \mathrm{v}_{200^{-}} ; \beta=$ parâmetros dos modelos a serem estimados; $\mathrm{Id}=$ idade

(anos) ; $\varepsilon=$ erro; $\ln =$ logaritmo neperiano.

\section{Determinação dos sortimentos}

Para quantificar o volume por sortimento de tora, utilizou-se a seguinte função de afilamento, ajustada a partir do modelo de Schöpfer (1966) para a espécie e região sob análise, considerando as diferentes idades:

$$
\begin{gathered}
\frac{\mathrm{di}}{\text { dap }}=1,27021-4,80717\left(\frac{\mathrm{h}_{\mathrm{i}}}{\mathrm{h}_{\mathrm{t}}}\right)+23,05823\left(\frac{\mathrm{h}_{\mathrm{i}}}{\mathrm{h}_{\mathrm{t}}}\right)^{2}-54,63023\left(\frac{\mathrm{h}_{\mathrm{i}}}{\mathrm{h}_{\mathrm{t}}}\right)^{3}+56,497071\left(\frac{\mathrm{h}_{\mathrm{i}}}{\mathrm{h}_{\mathrm{t}}}\right)^{4}-21,39555\left(\frac{\mathrm{h}_{\mathrm{i}}}{\mathrm{h}_{\mathrm{t}}}\right)^{5}+\varepsilon \\
\mathrm{R}^{2}=0,95, \mathrm{~S}_{\mathrm{yx}}=0,09
\end{gathered}
$$

em que: $d_{i}=$ diâmetros $(\mathrm{cm})$ medidos às alturas $h_{\mathrm{i}}$ ao longo do fuste; dap = diâmetro a $1,3 \mathrm{~m} ; \mathrm{h}_{\mathrm{t}}=$ altura total $(\mathrm{m})$.

\section{Análise Econômica}

Considerando a altura de toco de $0,10 \mathrm{~m}$ e toras de 2,5 $\mathrm{m}$ de comprimento, quantificou-se o volume por sortimento e, na sequência, seu valor de acordo com os preços praticados para Eucalyptus no Planalto Sul de Santa Catarina. Os sortimentos utilizados foram classificados de acordo com o diâmetro na ponta fina das toras, resultando em: 06 a $20 \mathrm{~cm}, \mathrm{R} \$ 5 \mathrm{~m}^{-3} ; 20-25 \mathrm{~cm}, \mathrm{R} \$ 20,00 \mathrm{~m}^{-3} ; 25-40 \mathrm{~cm}, \mathrm{R} \$ 75 \mathrm{~m}^{-3} ;>40 \mathrm{~cm}, \mathrm{R} \$ 125 \mathrm{~m}^{-3}$. Os custos de colheita e carregamento já foram descontados dos valores $\left(\mathrm{R} \$ 35 \mathrm{~m}^{-3}\right)$. Também, foram considerados os custos de implantação ( $\mathrm{R} \$ 5.000 \mathrm{ha}^{-1}$, ano 1), manutenção ( $\mathrm{R} \$ 500,00 \mathrm{ha}^{-1}$ ano $^{-1}$, nos primeiros dois anos) e administração ( $\mathrm{R} \$ 100 \mathrm{ha}^{-1} \mathrm{ano}^{-1}$ ), sendo iguais para todos os sítios. A análise econômica foi simplificada ao máximo, visando à obtenção de conclusões genéricas e robustas, e teve a construção de fluxos de caixa como base.

O regime de manejo considerado foi o mesmo para os três sítios, com três desbastes e corte raso aos 20 anos. Em função da falta de informações, foram consideradas as mesmas receitas líquidas dos desbastes nos três sítios (aproximações obtidas a partir de dados reais): $1^{\mathrm{o}}$ desbaste aos 4 anos (R $\left.\$ 250 \mathrm{ha}^{-1}\right), 2^{\circ}$ desbaste aos 6 anos $\left(\mathrm{R} \$ 500 \mathrm{ha}^{-1}\right)$ e $3^{\mathrm{o}}$ desbaste aos 9 anos $\left(\mathrm{R} \$ 1.500 \mathrm{ha}^{-1}\right)$.

Dessa forma, a única diferença no fluxo de caixa entre os sítios foi a receita obtida no corte raso aos 20 anos de idade. Para essa valoração, utilizou-se o método da árvore-modelo (CAMPOS; LEITE, 2013), tomando como base o valor monetário por árvore em cada um dos sítios. Esse valor foi obtido a partir da produção volumétrica e dos sortimentos de toras multiplicados pela densidade do povoamento no momento do corte raso, fixada em 200 árv. ha ${ }^{-1}$.

Como indicadores econômicos, foram utilizados o valor presente líquido (VPL), valor esperado da terra (VET) e taxa interna de retorno (TIR). A taxa mínima de atratividade (TMA) para o cálculo do VPL e VET foi de $5 \%$ ao ano (real, sem inflação). O valor da terra não foi considerado. 


\section{RESULTADOS}

\section{Classificação dos sítios}

Os coeficientes das equações ajustadas para o crescimento em altura dominante ao longo dos anos são apresentados na tabela 2. As estatísticas do ajuste e, principalmente, a análise gráfica dos resíduos indicaram que o modelo mais adequado para estimar o crescimento em altura dominante foi o exponencial.

Tabela 2. Coeficientes ajustados para o crescimento em altura dominante $\left(\mathrm{h}_{\mathrm{dom}}\right)$ e suas respectivas estatísticas.

Table 2. Coefficients fitted for dominant height growth $\left(\mathrm{h}_{\mathrm{dom}}\right)$ and their respective statistics.

\begin{tabular}{ccccccc}
\hline Modelos & $\beta_{0}$ & $\beta_{1}$ & $\beta_{2}$ & $\mathrm{R}^{2}$ & $\begin{array}{c}\mathrm{S}_{\mathrm{yx}} \\
(\mathrm{m})\end{array}$ & $\begin{array}{c}\mathrm{S}_{\mathrm{yx}} \\
\%\end{array}$ \\
\hline Polinomial & 3,4377 & 3,7563 & $-0,1026$ & 0,74 & 3,4 & 12,5 \\
Hiperbólico & 32,8996 & $-268,4552$ & & 0,57 & 4,3 & 15,8 \\
Potência & 2,1167 & 0,5494 & & 0,66 & 3,6 & 13,2 \\
Exponencial* & 3,8170 & $-4,1086$ & & 0,73 & 3,4 & 12,6
\end{tabular}

Em que: $\beta_{\mathrm{n}}=$ coeficientes estimados dos modelos; $\mathrm{R}^{2}=$ coeficiente de determinação; $\mathrm{S}_{\mathrm{yx}}=$ erro padrão da estimativa, em metros e em porcentagem. *modelo selecionado. figura 1 .

As curvas de crescimento em altura dominante, delimitando as classes de sítio, são apresentadas na

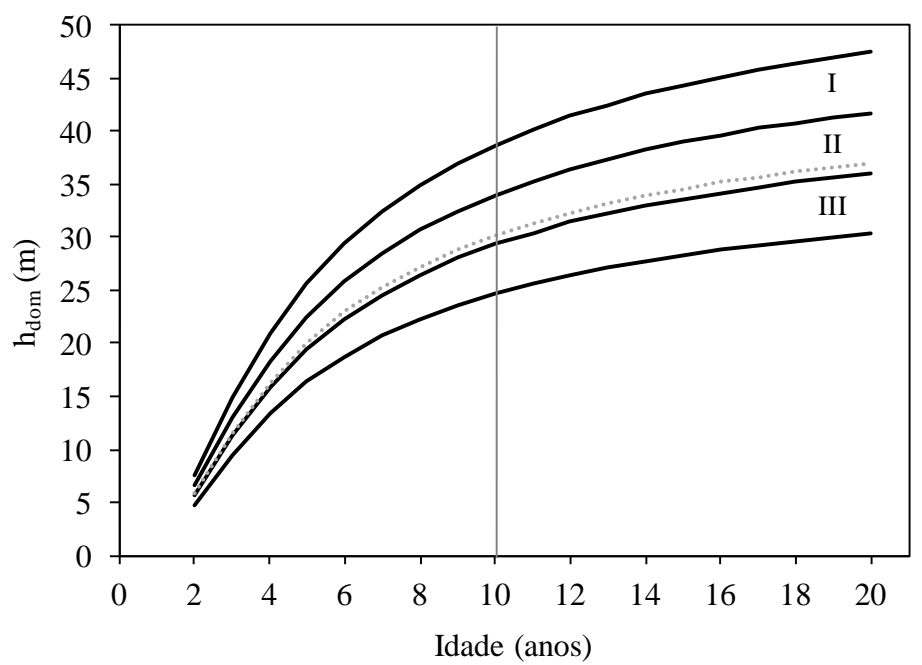

Figura 1. Crescimento médio em altura dominante para E. dunnii no Planalto Sul do Brasil (linha pontilhada). Curvas de crescimento em altura dominante $\left(\mathrm{h}_{\mathrm{dom}}\right)$ ao longo dos anos (linhas cheias), delimitando as classes de sítio para a espécie e região de análise. Idade índice: 10 anos. Classe I: 34 a 39 m; Classe II: 29 a 33,9 m; Classe III: 24 a 28,9 m.

Figure 1. Average dominant height growth for E. dunnii on the Highlands of Southern Brazil (dotted line). Growth curves of dominant height $\left(\mathrm{h}_{\mathrm{dom}}\right)$ over the years (full lines), which delimitated the site classes of the analyzed species and region. Age index: 10 years. Class I: 34 to $39 \mathrm{~m}$; Class II: 29 to 33,9 m; Class III: 24 to $28,9 \mathrm{~m}$.

Das 128 unidades amostrais avaliadas, 26 foram enquadradas na classe de sítio I, 56 na classe II e 46 na classe III. A curva pontilhada, que expressa o crescimento médio das árvores considerando todos os pares de dados utilizados no ajuste da equação, é visualizada no meio das curvas que delimitam a classe intermediária normalmente. Isso não ocorreu no presente estudo em virtude da amplitude da altura dominante (máxima e mínima) na idade índice, que apresentava valores proporcionalmente menores à curva guia ajustada. 


\section{Diâmetro médio $\left(d_{200}\right)$}

Os modelos foram testados para os pares de dados $\mathrm{d}_{200} \mathrm{x}$ idade de todos os sítios em conjunto, obtendose os coeficientes apresentados na tabela 3. Em função das estatísticas do ajuste e, principalmente, da análise gráfica dos resíduos, optou-se pelo modelo de potência para estimar o crescimento em diâmetro $\left(\mathrm{d}_{200}\right)$ ao longo dos anos para os diferentes sítios. Os pares de dados $\mathrm{d}_{200} \mathrm{x}$ idade foram então agrupados nas diferentes classes de sítio, possibilitando o ajuste de curvas de crescimento em diâmetro $\left(\mathrm{d}_{200}\right)$ por classe de sítio (Tabela 3$)$.

Tabela 3. Coeficientes obtidos no ajuste dos modelos para o crescimento médio em $\mathrm{d}_{200}$ para E. dunnii cultivados no Planalto Sul do Brasil e suas respectivas estatísticas de ajuste. Coeficientes obtidos no ajuste do modelo de potência para o crescimento médio em diâmetro $\left(\mathrm{d}_{200}\right)$ de E. dunnii nas diferentes classes de sítio e suas respectivas estatísticas de ajuste.

Table 3. Coefficients fitted for the average growth in $\mathrm{d}_{200}$ for $E$. dunnii grown on the Highlands of Southern Brazil and their respective statistics. Coefficients fitted for the power model for the diameter growth $\left(\mathrm{d}_{200}\right)$ of $E$. dunnii on the different site classes and their respective statistics.

\begin{tabular}{ccccccc}
\hline Modelos & $\beta_{0}$ & $\beta_{1}$ & $\beta_{2}$ & $\mathrm{R}^{2}$ & $\mathrm{~S}_{\mathrm{yx}}(\mathrm{cm})$ & $\mathrm{S}_{\mathrm{yx}} \%$ \\
\hline Polinomial & 5,3601 & 3,07266 & $-0,0691$ & 0,77 & 3,1 & 12,0 \\
Hiperbólico & 31,5565 & 31,5565 & & 0,51 & 4,6 & 17,6 \\
Potência* & 2,0568 & 0,5597 & & 0,75 & 3,3 & 12,6 \\
Exponencial & 3,7643 & $-3,9943$ & & 0,73 & 3,4 & 13,0 \\
\hline Classe de sítio & $\beta_{0}$ & $\beta_{1}$ & & $\mathrm{R}^{2}$ & $\mathrm{~S}_{\mathrm{yx}}(\mathrm{cm})$ & $\mathrm{S}_{\mathrm{yx}} \%$ \\
\hline I & 2,2326 & 0,5520 & & 0,66 & 3,6 & 11,9 \\
II & 2,0661 & 0,5493 & & 0,72 & 3,5 & 12,3 \\
III & 1,9972 & 0,5423 & & 0,61 & 2,8 & 12,7 \\
\hline
\end{tabular}

Em que: $\beta_{\mathrm{n}}=$ coeficientes estimados dos modelos; $\mathrm{R}^{2}=$ coeficiente de determinação; $\mathrm{S}_{\mathrm{yx}}$ erro padrão da estimativa em centímetros e porcentagem; *modelo selecionado.

A partir das equações ajustadas e apresentadas na tabela 3, foi possível traçar as curvas de crescimento em diâmetro $\left(\mathrm{d}_{200}\right)$ em função do aumento da idade para as diferentes classes de sítio (Figura 2).

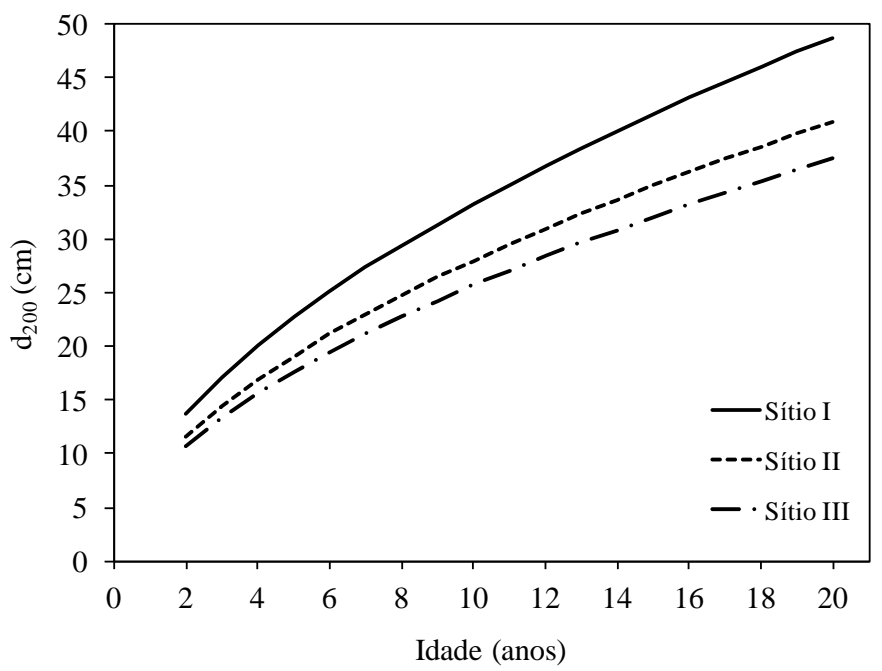

Figura 2. Curvas de crescimento em diâmetro $\left(\mathrm{d}_{200}\right)$ para E. dunnii nas diferentes classes de sítio no Planalto Sul do Brasil.

Figure 2. Growth curves in diameter $\left(\mathrm{d}_{200}\right)$ for E. dunnii on the different site classes on the Highlands of Southern Brazil. 
Volume ( $\left.\mathbf{v}_{\mathbf{2 0 0}}\right)$

De forma semelhante ao realizado com o diâmetro $\left(\mathrm{d}_{200}\right)$, os modelos foram ajustados para os pares de dados $v_{200} \mathrm{X}$ idade. Os coeficientes obtidos no ajuste são apresentados na tabela 4. A análise das estatísticas dos ajustes, incluindo a análise gráfica dos resíduos, indicou o modelo exponencial como o mais apropriado para estimar o volume médio das 200 árvores dominantes por hectare $\left(\mathrm{v}_{200}\right)$. A partir do mesmo agrupamento dos pares de dados por classe de sítio realizado para o $\mathrm{d}_{200}$, foram ajustadas curvas de crescimento em volume $\left(\mathrm{v}_{200}\right)$ por classe de sítio, utilizando-se o modelo exponencial (Tabela 4).

Tabela 4. Coeficientes obtidos no ajuste dos modelos para o crescimento médio em volume $\left(\mathrm{v}_{200}\right)$ de $E$. dunnii e suas respectivas estatísticas. Coeficientes obtidos no ajuste do modelo exponencial para o crescimento médio em volume $\left(\mathrm{v}_{200}\right)$ de $E$. dunnii nas diferentes classes de sítio e suas respectivas estatísticas de ajuste.

Table 4. Coefficients fitted for volume $\left(\mathrm{v}_{200}\right)$ growth models of E. dunnii with the respective statistics. Fitted coefficients for the exponential growth model in volume $\left(\mathrm{v}_{200}\right)$ of E. dunnii on the different site classes with the respective statistics.

\begin{tabular}{ccccccc}
\hline Modelos & $\beta_{0}$ & $\beta_{1}$ & $\beta_{2}$ & $\mathrm{R}^{2}$ & $\mathrm{Syx}\left(\mathrm{m}^{3}\right)$ & Syx $\%$ \\
\hline Polinomial & $-0,4918$ & 0,1634 & $-0,0023$ & 0,77 & 0,2 & 33,0 \\
Hiperbólico & 1,0953 & $-16,5096$ & & 0,41 & 0,3 & 48,5 \\
Potência & $-3,8039$ & 1,5578 & & 0,65 & 0,2 & 37,7 \\
Exponencial* & 0,9661 & $-11,2545$ & 0,92 & 0,2 & 33,0 \\
\hline Classe de sítio & $\beta_{0}$ & $\beta_{1}$ & $\mathrm{R}^{2}$ & $\mathrm{~S}_{\mathrm{yx}}\left(\mathrm{m}^{3}\right)$ & $\mathrm{S}_{\mathrm{yx}} \%$ \\
\hline I & 1,1488 & $-9,3650$ & 0,79 & 0,2 & 16,8 \\
II & 1,2004 & $-13,0446$ & 0,92 & 0,2 & 17,4 \\
III & 0,4473 & $-9,8990$ & 0,53 & 0,2 & 37,2 \\
\hline $\begin{array}{l}\text { Em que: } \beta_{\mathrm{n}}=\text { coeficientes estimados dos modelos; } \mathrm{R}^{2}=\text { coeficiente de determinação; } \\
\text { estimativa } \text { em metros e em porcentagem. }{ }^{*} \text { modelo selecionado. }\end{array}$
\end{tabular}

$\mathrm{O}$ ajuste do modelo exponencial para o crescimento em volume $\left(\mathrm{v}_{200}\right)$ nos diferentes sítios apresentou qualidades diferentes, dependo do sítio analisado. De qualquer forma, permitiu a construção de curvas de crescimento coerentes e comparáveis (Figura 3). Assim, obteve-se um melhor entendimento do desenvolvimento desta variável ao longo dos anos nos diferentes sítios.

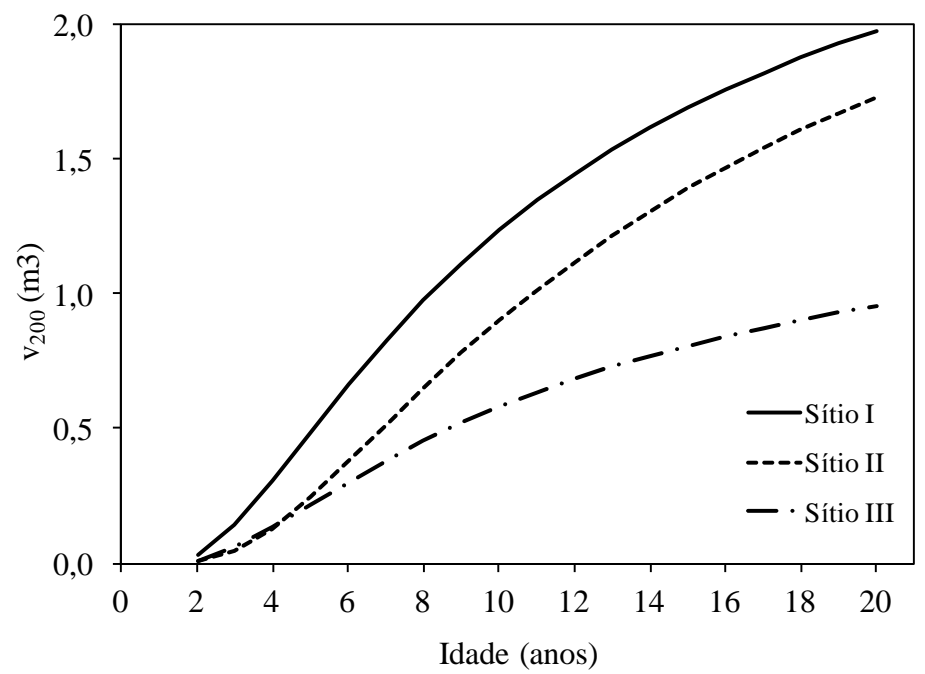

Figura 3. Curvas de crescimento em volume $\left(\mathrm{v}_{200}\right)$ a partir do modelo Exponencial ajustado para cada classe de sítio. Figure 3. Growth curves in volume $\left(\mathrm{v}_{200}\right)$ with aid of the fitted exponential models for each site class. 


\section{Desempenho econômico}

Devido à grande variação do crescimento observada, realizou-se uma análise econômica projetada dos povoamentos como reflexo do crescimento nas diferentes classes de sítios. A análise é considerada projetada em função da quantidade limitada de dados de povoamentos com mais de 15 anos, o que levou a algumas generalizações e aproximações.

O valor monetário da produção foi obtido a partir da valoração da árvore com as dimensões médias obtidas em cada sítio $\left(\mathrm{d}_{200} \mathrm{e} \mathrm{h}_{200}\right)$ (Tabela 5). Na sequência, esses valores foram extrapolados para um hectare.

Tabela 5. Estimativa de valor unitário para árvores de E. dunnii cultivados no Planalto Sul do Brasil com ciclo de produção de 20 anos, de acordo com as suas características dendrométricas.

Table 5. Estimated unit value for trees of E. dunnii grown on the Highlands of Southern Brazil within a production period of 20 years, according to their dendrometric characteristics.

\begin{tabular}{rccc}
\hline Classe de sítio & $\mathbf{d}_{\mathbf{2 0 0}}(\mathbf{c m})$ & $\mathbf{h}_{\mathbf{2 0 0}}(\mathbf{m})$ & Valor $_{\left(\mathbf{R} \text { \$ } \text { árv. }^{\mathbf{- 1}} \mathbf{)}\right.}$ \\
\hline I & 48,7 & 41,8 & 363 \\
II & 40,9 & 35,6 & 110 \\
III & 37,4 & 29,5 & 92 \\
\hline
\end{tabular}

Verificou-se que, aos 20 anos de idade, as árvores na classe de sítio I apresentavam um valor três vezes maior que as árvores do sítio II e quatro vezes superior aos obtidos no sítio III no momento do corte raso. A grande variação do valor médio das árvores colhidas no momento do corte raso possui impacto nos indicadores econômicos calculados para os diferentes sítios (Tabela 6).

Tabela 6. Taxa Interna de Retorno (TIR), Valor Presente Líquido (VPL) e Valor Esperado da Terra (VET) para povoamentos de E. dunnii cultivados no Planalto Sul do Brasil em diferentes sítios com três desbastes e corte raso aos 20 anos.

Table 6. Internal Rate of Return (IRR), Net Present Value (NPV) and Land Expectation Value (LEV) for $E$. dunnii grown on the Highlands of Southern Brazil on different site classes with three thinnings and clear cut at age of 20 years-old.

\begin{tabular}{cccc}
\hline \multirow{2}{*}{ Indicadores } & \multicolumn{3}{c}{ Classes de sítio } \\
\cline { 2 - 4 } & $\mathrm{I}$ & $\mathrm{II}$ & $\mathrm{III}$ \\
\hline TIR & $14,4 \%$ & $7,5 \%$ & $6,5 \%$ \\
VPL & $\mathrm{R} \$ 22.100$ & $\mathrm{R} \$ 3.000$ & $\mathrm{R} \$ 1.600$ \\
VET & $\mathrm{R} \$ 35.500$ & $\mathrm{R} \$ 4.800$ & $\mathrm{R} \$ 2.600$ \\
\hline
\end{tabular}

Valores arredondados para a centena mais próxima.

A maior TIR foi obtida no sítio I, sendo o dobro dos valores obtidos nos sítios II e III. Com relação ao VPL e VET, a diferença entre os sítios foi ainda maior. O valor obtido no sítio I foi 7 vezes superior ao obtido no sítio II e quase 14 vezes superior ao sítio III.

\section{DISCUSSÃO}

\section{Classificação dos sítios}

Observou-se que a altura dominante de povoamentos de $E$. dunnii pode variar de 25 a $39 \mathrm{~m}$ aos 10 anos de idade, demonstrando uma expressiva variação entre sítios. Alturas dominantes de 25 a 28,9 m aos 10 anos indicam um sítio de baixa produtividade (classe III), enquanto que o intervalo de 29 a 33,9 m representa um sítio intermediário (classe II). Ainda na idade índice, alturas dominantes entre 34 e $39 \mathrm{~m}$ indicam um sítio altamente produtivo (classe I) (Figura 1).

Lourenço (1996) avaliou o crescimento de E. dunnii no Planalto Norte de Santa Catarina e reportou alturas dominantes máximas de $25 \mathrm{~m}$ e mínimas de $14 \mathrm{~m}$ aos 7 anos de idade. Apesar de uma idade índice diferente à utilizada no presente estudo, pode-se afirmar que o trabalho desenvolvido pelo referido autor foi realizado em sítios intermediários a ruins. 


\section{Diâmetro médio $\left(\mathbf{d}_{200}\right)$}

Constatou-se que o diâmetro médio das 200 árvores mais grossas por hectare variou de 26 a $33 \mathrm{~cm}$ aos 10 anos de idade, sendo maior quanto melhor o sítio. A diferença do $\mathrm{d}_{200}$ aumentou com a idade. Aos 20 anos, a diferença entre os $\mathrm{d}_{200}$ obtidos nos sítios I e III foi de $12 \mathrm{~cm}$ ( $\mathrm{d}_{200}$ igual a $49 \mathrm{e} 37 \mathrm{~cm}$, respectivamente) (Figura 2).

Lourenço (1996) reportou povoamentos de E. dunnii com diâmetro de $24 \mathrm{~cm}$ aos 7 anos de idade. De acordo com os resultados obtidos no presente estudo, o povoamento avaliado pelo autor poderia ser enquadrado no sítio intermediário (classe II). Destaca-se, porém, que a comparação de diâmetros é frágil em função de não se saber exatamente o manejo empregado e, principalmente, pelo fato das curvas ajustadas no presente estudo serem referentes às 200 árvores mais grossas por hectare e não um diâmetro médio do povoamento, como é frequentemente utilizado.

Trugilho et al. (2004) observou povoamentos de E. dunnii com diâmetro médio de $27 \mathrm{~cm}$ aos 8 anos de idade e $34 \mathrm{~cm}$ aos 13 anos também no norte de Santa Catarina. Comparado com os resultados do presente trabalho, apesar de os critérios de comparação serem diferentes, conclui-se que os autores avaliaram sítios de produtividade intermediária a alta.

No Paraná, Reiner et al. (2012) avaliou povoamentos de E. dunnii aos 4 anos de idade com $14 \mathrm{~cm}$ de diâmetro, indicando se tratar de um sítio de baixa produtividade com valores inferiores aos estimados para a classe de sítio III.

\section{Volume ( $\left(\mathbf{v}_{200}\right)$}

Constatou-se que as árvores crescendo no sítio I atingem $1,2 \mathrm{~m}^{3}$ na idade índice de 10 anos e, aproximadamente, $2,0 \mathrm{~m}^{3}$ no final da rotação de 20 anos. No sítio II, o crescimento em volume ( $\left.\mathrm{v}_{200}\right)$ foi $25 \%$ inferior ao observado no sítio I aos 20 anos. No sítio III, observou-se uma grande redução de crescimento do volume em relação aos outros dois sítios. As árvores apresentaram $0,5 \mathrm{~m}^{3}$ aos 10 anos e menos de $1,0 \mathrm{~m}^{3}$ aos 20 anos, praticamente a metade do volume obtido no melhor sítio.

Verifica-se que a qualidade do sítio possui grande influência no volume dos indivíduos e, consequentemente, no potencial produtivo dos mesmos. Povoamentos estabelecidos em áreas cujo crescimento se assemelha ao observado na classe de sítio III apresentam incrementos médios anuais na ordem de $25 \mathrm{~m}^{3} \mathrm{ha}^{-1}$ $\mathrm{ano}^{-1}$ aos 10 anos de idade, enquanto que os da classe I ultrapassam os $50 \mathrm{~m}^{3} \mathrm{ha}^{-1}$ ano ${ }^{-1}$.

Ferrari et al. (2005) analisou o crescimento de E. dunnii no Centro Sul do Paraná e reportou um volume individual médio de $0,14 \mathrm{~m}^{3}$ aos 3 anos de idade e $0,45 \mathrm{~m}^{3}$ aos 7 anos, idade máxima avaliada pelo autor. Crescimento este equivalente ao observado na classe de sítio II do presente estudo. Já Reiner et al. (2015) relatou, para a mesma espécie aos 4 anos de idade no sudoeste do Paraná, volume individual médio de $0,1 \mathrm{~m}^{3}$, semelhante ao crescimento verificado na classe de sítio III da presente análise.

\section{Desempenho econômico}

Apesar da tendência óbvia de sítios produtivos resultarem maiores rentabilidades, surpreende a amplitude de variação entre os sítios. O fato do sítio I ter resultado VPL e VET 7 vezes superior ao sítio II e 14 vezes superior ao sítio III decorre da produção de toras com diâmetro superior a $40 \mathrm{~cm}$, o sortimento mais valioso, observada somente no sítio I. Na verdade, tomando como base a árvore com dimensões médias aos 20 anos de idade no sítio I, as cinco primeiras toras foram classificadas como $>40 \mathrm{~cm}$ na ponta fina, o que, certamente, contribuiu para o melhor resultado econômico.

Souza Jr. (2012) comparou o desempenho econômico de diferentes regimes de manejo para Pinus e Eucalyptus no Planalto Serrano Catarinense. Concluiu que o melhor resultado econômico foi obtido com o cultivo de Eucalyptus com desbastes e corte raso aos 20 anos, o que resultou em uma TIR de 11,7\% ao ano e um VET de $\mathrm{R} \$ 26.000 \mathrm{ha}^{-1}$ para uma taxa mínima de atratividade de $5 \%$, idêntica à utilizada no presente estudo. Chama a atenção o fato de o autor ter utilizado uma valoração para os sortimentos que não diferenciou toras com mais de $18 \mathrm{~cm}$ de diâmetro na ponta fina $\left(\mathrm{R} \$ 121,00 \mathrm{~m}^{-3}\right)$, o que difere substancialmente da presente análise. Mesmo assim, o regime de manejo com desbastes e ciclo longo superou a opção sem desbaste e corte raso aos 6 a 9 anos. Na verdade, essa última opção, denominada de ciclo curto pelo autor citado, apresentou o pior desempenho econômico entre os regimes de manejo avaliados para os dois gêneros. Os resultados apresentados por Souza Jr. (2012) permitem inferir que os dados utilizados em suas análises foram coletados em povoamento com produtividade intermediária a elevada, obtidos, possivelmente, de empresas cuja tecnologia de cultivo é bastante desenvolvida.

Constata-se que não é somente mais rentável produzir E. dunnii em sítios mais produtivos, mas também que o cultivo de E. dunnii pode não ser viável em sítios II e, principalmente, III. Apesar da necessidade de estudos adicionais, considerando a ótima adaptação de Pinus taeda L. à região de estudo e a menor necessidade tecnológica do seu cultivo, locais classificados como sítio II e III podem ter melhores resultados econômicos 
com o cultivo de Pinus, questionando, assim, a ideia de que o plantio de Eucalyptus resultaria sempre em melhores retornos econômicos em virtude de um ciclo potencialmente mais curto.

\section{CONCLUSÃO}

- Para E. dunnii no Planalto Sul Catarinense, foi possível determinar três classes de sítio com alturas dominantes $\left(\mathrm{h}_{\mathrm{dom}}\right)$, assim distribuídas na idade índice de 10 anos: Classe I: 34 a $39 \mathrm{~m}$; Classe II: 29 a 33,9 m; Classe III: 24 a 28,9 m.

- Há uma grande diferença no crescimento diamétrico e volumétrico das árvores nos diferentes sítios. Povoamentos com $\mathrm{d}_{200}$ de $30 \mathrm{~cm}$ podem ser obtidos já aos 9 anos de idade em sítios altamente produtivos. Entretanto, demoram entre 3 e 5 anos a mais para serem obtidos em sítios menos produtivos.

- A Taxa Interna de Retorno (TIR) de sítios com alto potencial produtivo é duas vezes maior que em sítios de menor produtividade. Valores Presente Líquido (VPL) e Esperados da Terra (VET) em sítio ótimo ( $\mathrm{h}_{\mathrm{dom}}$ de 34 a $39 \mathrm{~m}$ aos 10 anos) são, aproximadamente, 7 vezes superiores aos obtidos em sítio intermediário ( $\mathrm{h}_{\mathrm{dom}}$ de 29 a 33,9 m) e 14 vezes superiores aos obtidos em sítio ruim ( $\mathrm{h}_{\mathrm{dom}}$ de 24 a 28,9 m).

- Conclui-se que não é somente mais rentável produzir E. dunnii em sítios mais produtivos, mas que o cultivo de E. dunnii pode não ser viável em sítios menos produtivos.

\section{AGRADECIMENTOS}

Os autores são gratos à empresa Florestal Gateados Ltda. pela sessão dos dados e apoio ao estudo.

\section{REFERENCIAS}

ASSOCIAÇÃO BRASILEIRA DE PRODUTORES DE FLORESTAS PLANTADAS (ABRAF). Anuário estatístico da ABRAF 2013 ano base 2012. Brasília: ABRAF. 2013. 147 p.

ALFENAS, A. C.; ZAUZA, E. A. V.; MAFIA, R. G.; ASSIS, T. F. Clonagem e doenças do Eucalipto. 2. Ed. Viçosa, MG, Ed. UFV, 2009.

ARNOLD, R. J.; CLARKE, B.; LUO, J. Trials of cold-tolerant Eucalyptus species in cooler regions of South Central China. ACIAR Technical Report no. 57e, CSIRO, Canberra. 2004. 106 p.

CAMPOS, J. C. C.; LEITE, H. G. Mensuração Florestal: perguntas e respostas. 4. Ed. Viçosa, MG. Ed. UFV, 2013, $605 \mathrm{p}$.

DOBNER JR, M.; HIGA, A. R.; SEITZ, R. A. Efeito da cobertura de Pinus taeda L. na proteção contra geadas e no crescimento de plantas jovens de Eucalyptus dunnii Maiden. Floresta, Curitiba, v. 39, n. 4, p. 807-823, 2009.

FERRARI, M. P.; FERREIRA, C. A.; SILVA, H. D.; TREVISAN, R. Prognose do crescimento volumétrico individual de árvores de Eucalyptus, em povoamentos na Região Centro Sul. Bol. Pesq. Fl., Colombo, n. 51, p. 05-16, 2005.

GLENCROSS, K.; PALMER, G.; PELLETIER, M. C.; NICHOLS, J. D. Growth response to thinning in two subtropical hardwood species. Technical Report 217. Cooperative Research Centre for Forestry, Hobart. 2011.

HIGA, R. C. V. Avaliação e recuperação de Eucalyptus dunnii atingidos por geadas em Campo Tenente, Paraná. Tese (Doutorado em Ciências Florestais). UFPR. Curitiba, PR. 100 p. 1998.

HIGA, R. C. V.; HIGA, A. R.; TREVISAN, R.; SOUZA, M. V. R. Resistência e resiliência a geadas em Eucalyptus dunnii Maiden plantados em Campo do Tenente, PR. Bol. Pesq. Fl., Colombo, n. 40, p. 67-76. 2000.

HARWOOD, C.; BANDARA, K.; WASHUSEN, R.; NORTHWAY, R.; HENSON, M.; BOYTON, S. Variation in wood properties of plantation-grown Eucalyptus dunnii relevant to solid-wood products. Forest and Wood Products Research and Development Corporation, Project no. PN04.3003, Victoria, 40 p. 2005.

HERNANDEZ, J.; DEL PINO, A.; SALVO, L.; ARRARTE, G. Nutrient export and harvest residue decomposition patterns of a Eucalyptus dunnii Maiden plantation in temperate climate of Uruguay. For. Ecol. Manag. v. 258, n. 2: 92-99. doi: 10.1016/j.foreco.2009.03.050. 2009.

LOURENÇO, M. F. S. Características limitantes do solo no crescimento de Eucalyptus dunnii Maiden, em uma toposequencia na Região de Três Barras (SC). Dissertação (Mestrado em Engenharia Florestal). UFPR. Curitiba, PR. 140 p. 1996. 
MARCÓ, M.; WHITE, T. L. Genetic parameter estimates and genetics gains for Eucalyptus grandis and E. dunnii in Argentina. Forest Genetics, v. 9, n. 3, p. 205-215, 2002.

PALUDZYSZYN, E.F.; SANTOS, P. E. T. Considerações sobre o plantio de Eucalyptus dunnii no estado do Paraná. Colombo. Embrapa Floresta. Comunicado Técnico 141, 2005.

REINER, D. A.; SILVEIRA, R. E.; FERRONATO, M. L.; MENEGASSI, A. D. Desenvolvimento de Eucalyptus dunnii Maiden em diferentes espaçamentos de plantio em Pato Branco - Paraná. Anais $4^{\circ}$ Congresso Florestal Paranaense 2012.

ROCHA, M. P.; TRUGILHO, P. F. Qualidade de madeira serrada de Eucalyptus dunnii em função do método de desdobro e condição de umidade. Cerne, Lavras, v. 12, n. 4, p. 314-321. 2006.

SCOLFORO. J. R. S. Biometria Florestal- módulo 3: métodos para classificação de sítios florestais. Lavras: UFLA/FAEPE. 1997. 151 p.

SOUZA JR., J. O. Análise econômica em plantios de Pinus e Eucalyptus no Planalto Serrano Catarinense. (Mestrado em Engenharia Florestal). UFPR. Curitiba, PR. 2012. 173 p.

TRUGILHO, P. F.; IWAKIRI, S.; ROCHA, M. P.; MATOS, J. L. M.; SALDANHA, L. K. Efeitos da idade e classe diamétrica na deformação residual longitudinal em árvores de Eucalyptus dunnii Maiden. Árvore, Viçosa, v. 28, n. 5, p. 725-731, 2004.

ZANON, M. L. B.; FINGER, C. A. G.; SCHNEIDER. P. R.; KLEIN, J. E. M.; COELHO, M. C. B. Funções para descrever a relação altura diâmetro de Ecalyptus dunnii Maiden. Ciência Rural, Santa Maria, v. 26, n. 1, p. 8790, 1996. 